\title{
Correction to: Artificial intelligence: a critical review of current applications in pancreatic imaging
}

\author{
Maxime Barat ${ }^{1,2} \cdot$ Guillaume Chassagnon $^{1,2} \cdot$ Anthony Dohan $^{1,2} \cdot$ Sébastien Gaujoux ${ }^{2,3} \cdot$ Romain Coriat $^{2,4}$. \\ Christine Hoeffel $^{5}$. Christophe Cassinotto ${ }^{6}$. Philippe Soyer ${ }^{1,2}$ (])
}

Published online: 10 March 2021

(c) Japan Radiological Society 2021

Correction to: Japanese Journal of Radiology

https://doi.org/10.1007/s11604-021-01098-5

In the original publication, the caption of Figure 1 and Figure 2 are interchanged. In this Correction, Figs. 1 and 2 are included with correct captions.

The original article has been corrected.

Publisher's Note Springer Nature remains neutral with regard to jurisdictional claims in published maps and institutional affiliations.

The original article can be found online at https://doi.org/10.1007/ s11604-021-01098-5.

Philippe Soyer

philippe.soyer@lrb.aphp.fr

1 Department of Radiology, Hopital Cochin, Assistance Publique-Hopitaux de Paris, 27 Rue du Faubourg Saint-Jacques, Paris, France

2 Université de Paris, Descartes-Paris 5, 75006 Paris, France

3 Department of Abdominal Surgery, Hopital Cochin, Assistance Publique-Hopitaux de Paris, 75014 Paris, France

4 Department of Gastroenterology, Hopital Cochin, Assistance Publique-Hopitaux de Paris, 75014 Paris, France

5 Department of Radiology, Robert Debré Hospital, 51092 Reims, France

6 Department of Radiology, CHU Montpellier, University of Montpellier, Saint-Éloi Hospital, 34000 Montpellier, France 


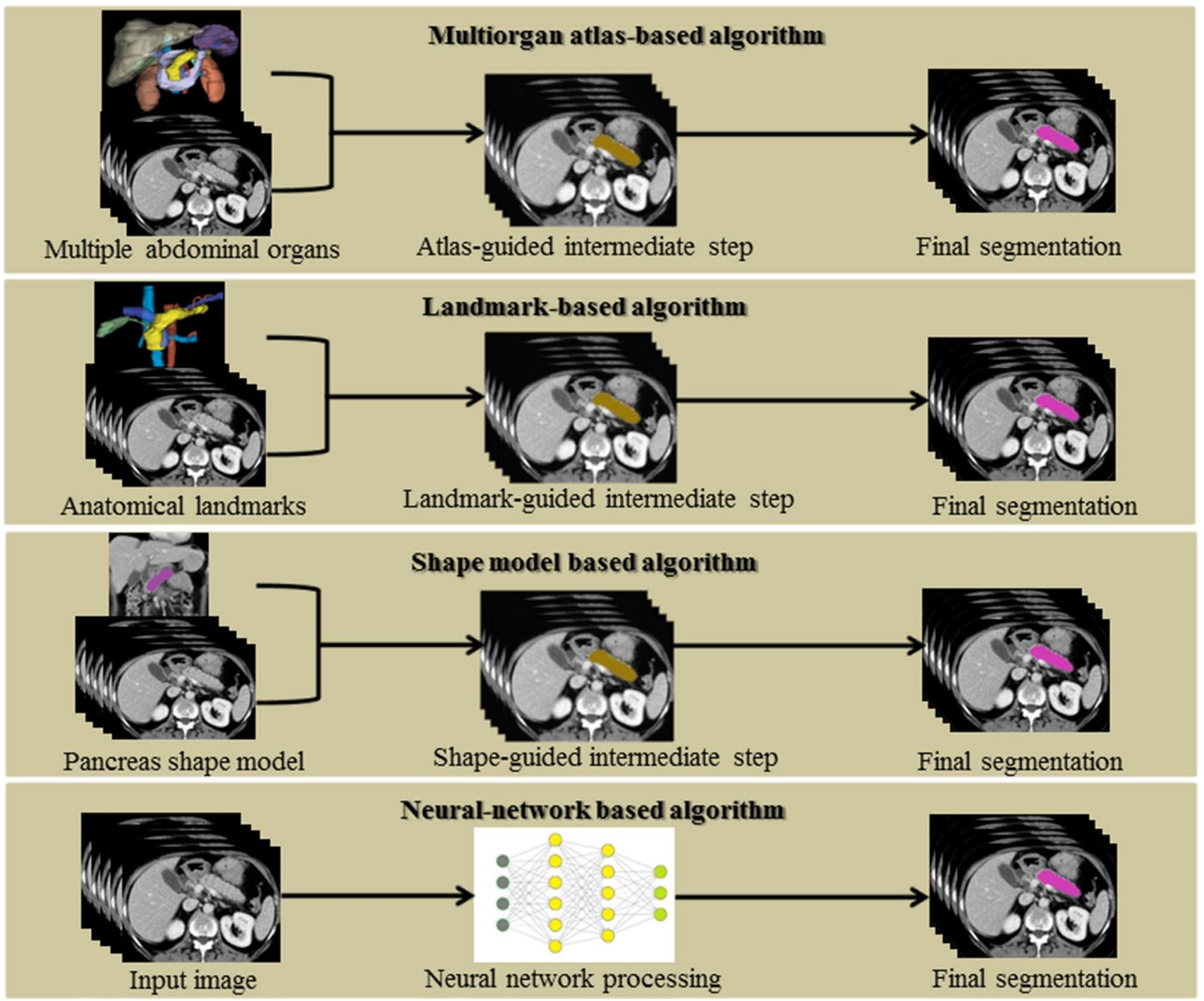

Fig. 1 The classification of the four algorithms used for pancreas segmentation in artificial intelligence 


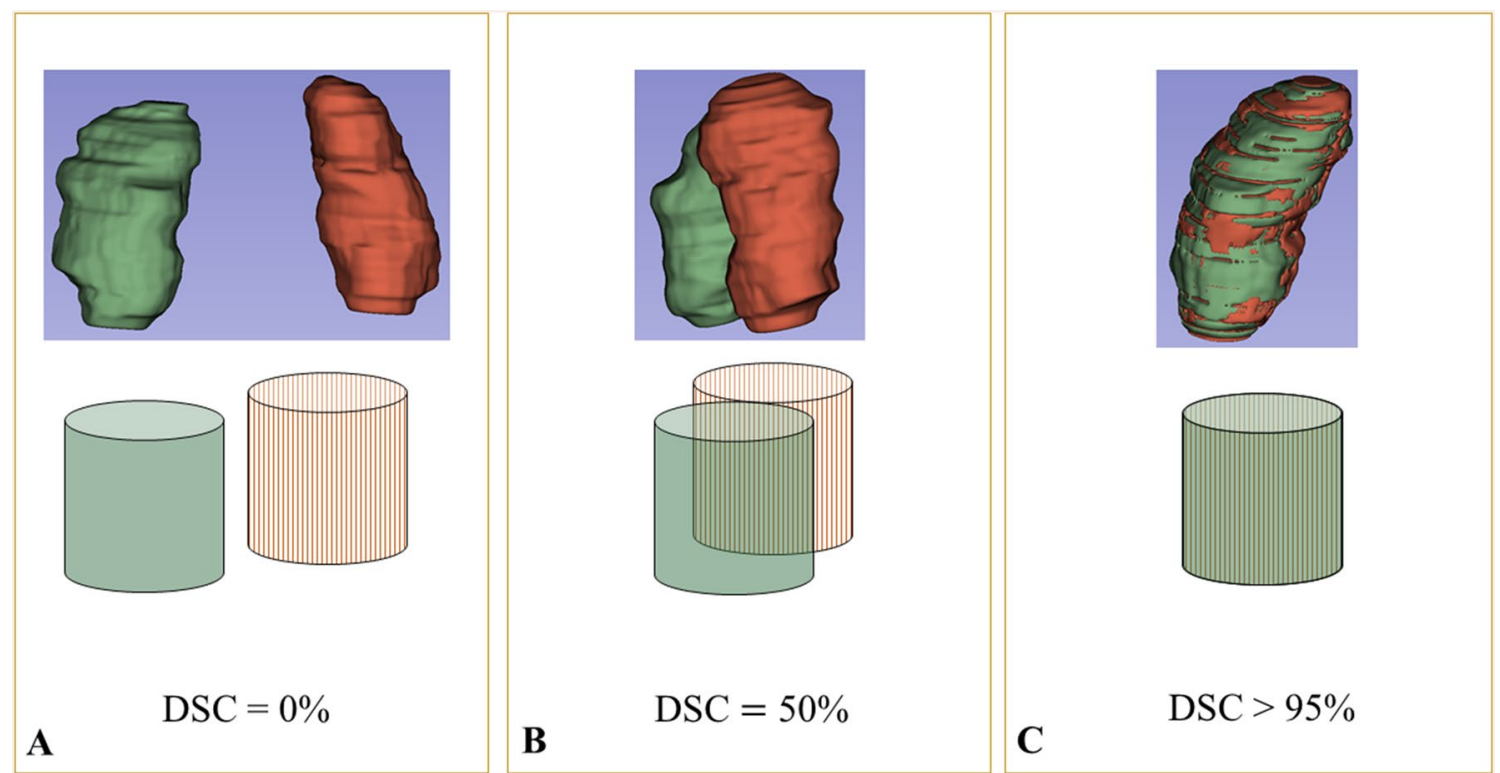

Fig. 2 Graph shows the basic principle of Dice similarity coefficient (DSC) calculation. Increasing DSC indicates increasing similarity (i.e., increasing spatial overlap) between ground truth (manual segmentation of a given object) and automated probabilistic segmenta- tion of the same object by the artificial intelligence algorithm. In a, there is no overlap yielding a $0 \%$ DSC. In $\mathbf{b}$, there is partial overlap (50\%), yielding a 50\% DSC. In c, there is complete overlap, yielding $100 \%$ DSC 